\title{
Feasibility of intensity-modulated radiotherapy combined with gemcitabine and S-1 for patients with pancreatic cancer
}

\author{
NORIFUMI KENNOKI ${ }^{1}$, HIDETSUGU NAKAYAMA ${ }^{1}$, YUICHI NAGAKAWA ${ }^{2}$, YUICHI HOSOKAWA ${ }^{2}$, \\ TOMOHIRO ITONAGA $^{1}$, YU TAJIMA ${ }^{1}$, SACHICA SHIRAISHI $^{1}$, RYUJI MIKAMI $^{1}$, \\ AKIHIKO TSUCHIDA ${ }^{2}$ and KOICHI TOKUUYE ${ }^{1}$ \\ ${ }^{1}$ Department of Radiology and ${ }^{2}$ Third Department of Surgery, Tokyo Medical University, Shinjyuku, Tokyo 160-0023, Japan
}

Received November 27, 2014; Accepted October 22, 2015

DOI: $10.3892 / \mathrm{mco} .2015 .664$

\begin{abstract}
The aim of the present study was to establish whether intensity-modulated radiotherapy (IMRT) with concurrent gemcitabine and S-1 is a feasible treatment option for patients with locally advanced pancreatic ductal adenocarcinoma. Patients with pancreatic ductal adenocarcinoma were prospectively enrolled. An IMRT dose of $50.4 \mathrm{~Gy}$ in 28 fractions with concurrent gemcitabine at a dose of $600 \mathrm{mg} / \mathrm{m}^{2}$ and $\mathrm{S}-1$ at a dose of $60 \mathrm{mg}$ were administrated. Adverse events and associated dosimetric factors were assessed. Between February 2012 and January 2014, 17 patients with borderline resectable and 4 with unresectable pancreatic cancer were enrolled. None of the patients experienced grade 3 or worse nausea and vomiting. The planning target volume ( $\geq 200$ vs. $<200 \mathrm{ml}$ ) was a statistically significant predictive factor for neutrocytopenia ( $\geq 500$ vs. 500/ $\mu 1, \mathrm{P}=0.02$ ). Concurrent IMRT with gemcitabine and S-1 for patients with locally advanced pancreatic cancer is feasible, with tolerable hematological toxicities and low gastrointestinal toxicities.
\end{abstract}

\section{Introduction}

Pancreatic cancer is the fifth leading cause of cancer-related fatalities in Japan (1), with an incidence rate almost identical to the crude death rate, reflecting the intractable nature of this disease (2). Finding an effective therapeutic approach for pancreatic cancer remains challenging. A Japanese randomized trial compared surgery with chemoradiotherapy for locally resectable invasive pancreatic cancer, and its findings suggested that surgery remains the only potentially curative treatment (3), and subsequently, only if there is a negative surgical margin (4). There has been much debate as to whether

Correspondence to: Dr Hidetsugu Nakayama, Department of Radiology, Tokyo Medical University, 6-7-1 Nishi-Shinjyuku, Shinjyuku, Tokyo 160-0023, Japan

E-mail: hnakayam@tokyo-med.ac.jp

Key words: pancreatic cancer, intensity-modulated radiotherapy, chemoradiotherapy, preoperative the use of preoperative chemoradiotherapy can help achieve a clear margin and hence prolong survival (5).

In locally advanced and metastatic pancreatic cancer, gemcitabine and oral fluoropyrimidines S-1 (GS) resulted in a better response compared to gemcitabine alone (6), although, as expected, there were correspondingly worse hematological and gastrointestinal (GI) toxicities. To improve the resection rate, Motoi et al (7) conducted a phase II study of neoadjuvant GS for resectable and borderline resectable pancreatic cancer. They concluded that neoadjuvant GS was well tolerated and did not reduce operable rates.

Preoperative radiotherapy of pancreatic cancer is required to achieve a clear surgical margin, and although the addition of GS may further improve the chances of a clear margin, this is also expected to increase toxicity. A phase II study of prospective preoperative chemoradiotherapy for patients with locally advanced pancreatic cancer was conducted to improve the resection rate. This included intensity-modified radiotherapy (IMRT) in an attempt to reduce toxicity. The present study reports the resulting acute toxicities and feasibility of IMRT with GS for patients with locally advanced pancreatic adenocarcinoma.

\section{Materials and methods}

Patients. The ethical board of Tokyo Medical University (Tokyo, Japan) approved this prospective study (UMIN000008614). All the patients provided written informed consent. Eligibility criteria were as follows: Histologically or cytologically proven pancreatic ductal adenocarcinoma; borderline resectable or locally advanced unresectable pancreatic cancer diagnosed by contrast enhanced abdominal computed tomography (CT); $\geq 20$ years; no distant metastases or peritoneum dissemination found using thorax, abdomen and pelvis CT or magnetic resonance imaging (MRI) with contrast media and a 18-fluorodeoxyglucose-PET (FDG-PET) scan; Zubroad performance status of 0,1 or 2 ; no prior radiotherapy to the upper abdomen; and patients should be able to comprehend their disease and this study. The exclusion criteria were as follows: Life expectancy of $\leq 4$ months, any co-morbidities, women of child-bearing age, expectant or nursing women, a history of severe drug allergy and synchronous active cancer. 
Radiotherapy. All the patients were positioned within a fixed customized body shell to restrain respiratory motions, following which a CT image was obtained in the expiratory and inspiratory phases under free respiration. The gross tumor volume (GTV) included the pancreatic tumor and any enlarged lymph node $>1 \mathrm{~cm}$ in diameter. The clinical target volume (CTV) included the celiac and para-aortic lymph node basins, and the GTV. Internal target volume (ITV) was determined from CT images obtained in the expiratory and inspiratory phases. Organs at risk were determined to be the liver, stomach, duodenum, colon and kidney, as well as the spinal cord, and were delineated on the CT image of the expiratory phase. Planning target volume (PTV) was defined as the ITV with a $0.5-1 \mathrm{~cm}$ margin in all directions. IMRT was used to generate optimized treatment plans for each patient. Compensated filters were used in five non-coplanar directions to deliver IMRT treatment. Typically, the gantry angles were $72^{\circ}, 144^{\circ}, 216^{\circ}$ and $288^{\circ}$ with a couch angle of $0^{\circ}$, and $340^{\circ}$ with a couch angle of $90^{\circ}$. A prescribed dose of $50.4 \mathrm{~Gy}$ administered in 28 fractions was determined to be sufficient to cover $95 \%$ of the PTV.

Chemotherapy and surgery. Three weeks before chemoradiotherapy, all the patients received one cycle of gemcitabine at a dose of $1,000 \mathrm{mg} / \mathrm{m}^{2}$ in the first and second weeks. Gemcitabine at a dose of $600 \mathrm{mg} / \mathrm{m}^{2}$ (days 1, 8, 22 and 29) and S-1 at a dose of $60 \mathrm{mg}$ (days 1 through 14 and 22 through 36) were administrated with concurrent IMRT. When grade 4 or worse neutrocytopenia or thrombocytopenia occurred, chemotherapy was stopped for at least one week. Four weeks after the completion of chemoradiotherapy, CT and FDG-PET were used to evaluate operability, and surgery was performed when it was considered possible.

Stratification. All the patients were stratified into two groups based on CT findings at the entry of study. Borderline resectable pancreatic cancer was defined by the encasement of the gastroduodenal artery or abutment of the proper hepatic artery or superior mesenteric artery within $180^{\circ}$. Tumors that had invaded the hepatic or superior mesenteric vein were also considered to be borderline. Unresectable pancreatic cancer was defined by the abutment of the hepatic or superior mesenteric artery $>180^{\circ}$ or invasion of the aorta, and/or by an occlusion of the portal or superior mesenteric vein, which could not be reconstructed.

Assessments. Physical examination and blood analysis (red blood cell, white blood cell and platelet counts) and serum biochemistry (aspartate transaminase, alanine aminotransaminase, lactate dehydrogenase, total bilirubin, creatinine and albumin) was performed weekly from the initiation of radiotherapy until two weeks after completion of chemoradiotherapy. Adverse events were assessed according to common terminology criteria for adverse events (CTCAE, version 4.0; http://evs.nci.nih.gov/ftp1/CTCAE/CTCAE_4.03_2010-06-14_ QuickReference_5x7.pdf).

Statistics. Categorical variables were analyzed using the $\chi^{2}$ test and Fisher's exact test, and the correlation between continuous variables was analyzed using Spearman's rank test with STATA version 12 software (Stata Co., Texas, USA). $\mathrm{P}<0.05$ was considered to indicate a statistically significant difference.
Table I. Patient characteristics $(n=21)$.

\begin{tabular}{lc}
\hline Characteristics & Patients \\
\hline Age, median years (range) & $68(36-81)$ \\
Gender, $\mathrm{n}$ & 13 \\
Male & 8 \\
Female & \\
T stage, $\mathrm{n}$ & 21 \\
T4 & \\
N stage, $\mathrm{n}$ & 13 \\
N0 & 7 \\
N1 & 1 \\
N2 & \\
UICC stage, $\mathrm{n}$ & 20 \\
IVa & 1 \\
IVb & \\
Surgical status, $\mathrm{n}$ & 17 \\
Bordeline resectable & 4 \\
Unresectable & \\
Performance status, $\mathrm{n}$ & \\
0 & \\
Tumor location, $\mathrm{n}$ & \\
Head & \\
Body & \\
\hline
\end{tabular}

\section{Results}

Patient characteristics. Between February 2012 and January 2014, 21 patients were enrolled in the study (Table I), 17 of whom were diagnosed with borderline resectable pancreatic cancer and 4 with unresectable pancreatic cancer. Chemoradiotherapy had to be discontinued in one case due to a biliary infection. Thus, a total of 20 patients completed chemoradiotherapy, following which, 1 patient refused surgery. $\mathrm{CT}$ and/or PET revealed that 6 patients had metastases. Two of the 4 patients diagnosed with unresectable pancreatic cancer were still considered to have an unresectable tumor, and the remaining 11 patients underwent surgery.

Adverse events reported. Adverse events are summarized in Table II. In total, 13 (62\%) and 10 (48\%) patients experienced grade 3 or worse toxicities in white blood cells and neutrophils, respectively. None had fever with grade 3 or grade 4 neutrocytopenia, and only 1 experienced grade 3 or worse thrombocytopenia. None experienced grade 3 or worse vomiting or nausea. One patient, who had previously undergone a biliary decompression using a stent, suffered a grade 5 biliary infection following the initiation of chemoradiotherapy. Radiotherapy for this patient was discontinued after a total dose of 21.6 Gy in 12 fractions had been administered, due to a fever caused by the biliary infection 2 weeks after chemoradiotherapy initiation. The patient succumbed to sepsis 62 days after the start of chemoradiotherapy. All the other patients underwent 2 cycles of GS chemotherapy and 
Table II. Hematological and non-hematological toxicities $(n=21)$.

\begin{tabular}{lccccc}
\hline Toxicities & Grade 1, n (\%) & Grade 2, n (\%) & Grade 3, n (\%) & Grade 4, n (\%) & Grade 5, n (\%) \\
\hline White blood cell decrease & $2(10)$ & $6(29)$ & $12(57)$ & $1(5)$ & $0(0)$ \\
Neutorophil count decrease & $5(24)$ & $6(29)$ & $7(33)$ & $3(14)$ & $0(0)$ \\
Platelet count decrease & $15(71)$ & $4(19)$ & $1(5)$ & $1(5)$ & $0(0)$ \\
Nausea & $8(38)$ & $2(10)$ & $0(0)$ & $0(0)$ & $0(0)$ \\
Biliary tract infection & $0(0)$ & $0(0)$ & $0(0)$ & $0(0)$ & $1(5)$ \\
\hline
\end{tabular}

Table III. Difference of dose parametric factors between the toxicity of nausea.

\begin{tabular}{lccc}
\hline & \multicolumn{2}{c}{$\begin{array}{c}\text { Dosimetric parameters, } \\
\text { mean } \mathrm{ml} \pm \text { standard deviation }\end{array}$} & \\
\cline { 2 - 3 } & Grade 0 & Grade 1 or 2 & \\
Factors & nausea $(\mathrm{n}=11)$ & nausea $(\mathrm{n}=10)$ & P-value \\
\hline PTV & $204.1 \pm 122.8$ & $191.0 \pm 110.6$ & NS \\
Liver $\mathrm{V}_{10}$ & $14.9 \pm 10.8$ & $16.3 \pm 20.5$ & $\mathrm{NS}$ \\
Liver $\mathrm{V}_{20}$ & $5.2 \pm 4.3$ & $6.0 \pm 10.1$ & $\mathrm{NS}$ \\
Liver $\mathrm{V}_{30}$ & $1.1 \pm 1.0$ & $1.8 \pm 3.9$ & $\mathrm{NS}$ \\
Stomach $\mathrm{V}_{20}$ & $29.1 \pm 35.3$ & $35.8 \pm 22.1$ & $\mathrm{NS}$ \\
Stomach $\mathrm{V}_{30}$ & $13.5 \pm 27.3$ & $15.0 \pm 9.3$ & $\mathrm{NS}$ \\
Stomach $\mathrm{V}_{40}$ & $10.2 \pm 25.4$ & $5.9 \pm 4.2$ & $\mathrm{NS}$ \\
Duodenum $\mathrm{V}_{20}$ & $49.2 \pm 24.3$ & $47.7 \pm 26.0$ & $\mathrm{NS}$ \\
Duodenum $\mathrm{V}_{30}$ & $36.9 \pm 27.7$ & $34.3 \pm 27.2$ & $\mathrm{NS}$ \\
Duodenum $\mathrm{V}_{40}$ & $25.1 \pm 26.0$ & $19.6 \pm 20.9$ & $\mathrm{NS}$ \\
\hline
\end{tabular}

PTV, planned target volume; NS, not significant.

radiotherapy on an outpatient basis, except during gemcitabine administration. The size of the PTV ( $\geq 200$ vs. $<200 \mathrm{ml}$ ) was a statistically significant factor associated with neutrocytopenia ( $\geq 500$ vs. $<500 / \mu 1, \mathrm{P}=0.02$ ) and leucocytopenia ( $\geq 1,500$ vs. $<1,500 / \mu 1, \mathrm{P}<0.01)$, and was also correlated with a nadir platelet count $(\mathrm{P}=0.04)$. Table III shows the differences in dosimetric parameters for the organs at risk between patients with grade 0 and patients with grade 1 or 2 toxicity. There were no apparent associations between non-hematological toxicities and dosimetric parameters.

\section{Discussion}

Nucleoside analogs of 5-fluorouracil (5-FU) and gemcitabine are potent radiosensitizers, and the concomitant use of radiotherapy with 5-FU (8) and gemcitabine (9) has been shown to improve the survival of patients with locally advanced pancreatic cancer compared to radiotherapy alone. The aim of the present study was to establish whether concomitant radiotherapy and GS chemotherapy can convert unresectable (such as locally advanced to borderline resectable) cancer to resectable cancer, allowing tumors with a positive surgical margin to be treated as if they effectively had a negative margin. Based on the data of a phase III study conducted in Taiwan and Japan, S-1 for locally advanced and metastatic pancreatic cancer resulted in equivalent overall survival to GS (6). However, GS resulted in a significantly better response rate compared to gemcitabine alone $(\mathrm{P}<0.001)$. GS also resulted in significantly better progression-free survival compared to gemcitabine alone [hazard ratio $(\mathrm{HR})=0.66,95 \%$ confidence interval, 0.54-0.81, $\mathrm{P}<0.001$ ], and in subgroup analysis GS for locally advanced cancer produced better overall survival compared to gemcitabine alone $(\mathrm{HR}=0.67,95 \%$ confidence interval 0.46-0.99). Therefore, if toxicities are tolerable, GS appears to be a suitable addition to concurrent radiotherapy in the preoperative setting.

The Taiwan and Japan trial used $1,000 \mathrm{mg} / \mathrm{m}^{2}$ of gemcitabine and S-1 at a dose of 60, 80 and $100 \mathrm{mg}$ per patient, which was the same as the dose used in the present study. The incidence of grade 3 or worse leucocytopenia or neutrocytopenia was 68 and $38 \%$ in the Taiwan and Japan study, respectively, which were comparable to the frequency observed in the present study. The Taiwan and Japan study also found that grade 3 or worse nausea and vomiting was suffered by $5 \%$ of patients. These findings indicate that the toxicity of combined GS and IMRT is comparable to that of GS alone.

In the present study, 1 patient succumbed as a result of biliary sepsis. The patient experienced a $38.0^{\circ} \mathrm{C}$ fever 2 weeks after the initiation of chemoradiotherapy, and radiotherapy had to be discontinued one week after this. Pisters et al (10) reported that $13 \%$ of patients had a stent-related complication 12 weeks after the start of radiotherapy, although the majority of cases were not apparent by 6 weeks. The study concluded that a biliary stent did not increase the risk of infection in patients with potentially resectable pancreatic cancer, and that radiotherapy did not exacerbate biliary infection.

Krishnan et al (11) retrospectively compared the survival of patients treated using gemcitabine-based induction chemotherapy of a median 2.5 months followed by chemoradiotherapy with initial chemoradiotherapy, and found that this extended the survival of patients with locally advanced pancreatic cancer when induction chemotherapy was followed by chemoradiotherapy. This may have been because patients with rapid distant progression did not undergo induction chemotherapy. In the present study, one third of patients were spared ineffective surgery as distant metastases were detected during induction therapy, which is an advantage of this treatment strategy.

Loehrer et al (9) reported the toxicities that arose in 34 patients enrolled on the Eastern Cooperative Oncology Group (ECOG) phase III study, in which a radiation dose of 
50.4 Gy administered in 28 fractions was combined with a gemcitabine dose of $600 \mathrm{mg} / \mathrm{m}^{2}$. Patients treated using IMRT were excluded from this study to ensure a homogenous cohort. The study reported that grade 3 or worse leucocytopenia and neutrocytopenia were suffered by 33 and $21 \%$ of these patients, respectively. In the present study, oral S-1 was added to the treatment regimen, and the incidence of leucocytopenia and neutrocytopenia increased to 62 and 47\%, respectively, which were higher than reported in the ECOG study, although none had fever with grade 3 or 4 neutrocytopenia. By contrast, the incidence of grade 3 or worse nausea and vomiting was lower compared to the ECOG study. It is noteworthy that the GTV of the pancreatic tumor and adjacent lymph node was irradiated using anterior-posterior opposite projections in the ECOG study. We speculate that IMRT may reduce the irradiated volume and consequently non-hematological toxicities.

Yovino et al (12) reported that the incidence of treatment-related toxicity of IMRT combined with 5-FU for postoperative pancreatic cancer was significantly lower compared to in the RTOG 97-04 trial. There were no cases of grade 3 or worse nausea and vomiting, which concurs with the findings reported in the present study. In another trial, Ben-Josef et al (13) reported the toxicities arising following IMRT with a median dose of 54 Gy combined with capecitabine for 15 patients. The study also found no grade 3 or worse nausea and vomiting toxicities. These previous studies, together with the present, suggest that toxicity, particularly nausea and vomiting, can be reduced using IMRT instead of conventional radiotherapy for pancreatic cancer.

Concerning the dose parametric factors associated with acute toxicities, Murphy et al (14) reported the outcomes of concurrent three-dimensional conformal radiotherapy with gemcitabine, in which a median dose of 36 Gy was administered in 15 fractions with $1,000 \mathrm{mg} / \mathrm{m}^{2}$ gemcitabine (days 1 , 8 and 15) to 74 patients with unresectable pancreatic cancer. A total of $22 \%$ of these patients suffered grade 3 or worse GI toxicities, and there was a substantial difference in GI toxicities amongst patients with a PTV $\geq 260 \mathrm{ml}$ and those with a PTV $<260 \mathrm{ml}$. Nakamura et al (15) reported that GI toxicity was associated with the stomach $\mathrm{V}_{50}$ in gemcitabine-based chemoradiotherapy. In their study, a gemcitabine dose of $250 \mathrm{mg} / \mathrm{m}^{2}$ was administrated weekly together with three-dimensional conformal radiotherapy, and GTV was defined as the primary tumor plus the metastatic lymph nodes. Patients with a V50 $<16 \mathrm{ml}$ of the stomach had significantly fewer GI toxicities than those with a V50 of $\geq 16 \mathrm{ml}$. The irradiated volumes for organs at risk in this study were much smaller than those in the studies by Murphy et al (14) and Nakamura et al, which may explain why there were fewer GI toxicities.

Nakamura et al (15) reported that only $5 \%$ of patients had peripancreatic lymph node recurrence even though the PTV was limited to the primary tumor with a $1 \mathrm{~cm}$ margin that did not include the draining lymph node. The study concluded that the use of an irradiated volume encompassing only the GTV was not the cause of marginal failure, and resulted in fewer toxicities. The same methodology as Murphy et al (14) was used in the present study, and a similar reduction in toxicity was observed.

In conclusion, the present results indicate that concurrent IMRT with GS for patients with locally advanced pancreatic cancer is feasible and reduces toxicity, particularly nausea and vomiting, and its use is recommended for these patients.

\section{References}

1. Matsuno S, Egawa S, Fukuyama S, Motoi F, Sunamura M, Isaji S, Imaizumi T, Okada S, Kato H, Suda K, et al: Pancreatic cancer registry in Japan: 20 years of experience. Pancreas 28: 219-230, 2004.

2. Siegel R, Ma J, Zou Z and Jemal A: Cancer statistics, 2014. CA Cancer J Clin 64: 9-29, 2014

3. Imamura M, Doi R, Imaizumi T, Funakoshi A, Wakasugi $H$, Sunamura M, Ogata Y, Hishinuma S, Asano T, Aikou T, et al: A randomized multicenter trial comparing resection and radiochemotherapy for resectable locally invasive pancreatic cancer. Surgery 136: 1003-1011, 2004.

4. Bilimoria KY, Talamonti MS, Sener SF, Bilimoria MM, Stewart AK, Winchester DP, Ko CY and Bentrem DJ: Effect of hospital volume on margin status after pancreaticoduodenectomy for cancer. J Am Coll Surg 207: 510-519, 2008.

5. Klautke G and Brunner TB: Radiotherapy in pancreatic cancer. Strahlenther Onkol 184: 557-564, 2008.

6. Ueno H, Ioka T, Ikeda M, Ohkawa S, Yanagimoto H, Boku N, Fukutomi A, Sugimori K, Baba H, Yamao K, et al: Randomized phase III study of gemcitabine plus S-1, S-1 alone, or gemcitabine alone in patients with locally advanced and metastatic pancreatic cancer in Japan and Taiwan: GEST study. J Clin Oncol 31: 1640-1648, 2013

7. Motoi F, Ishida K, Fujishima F, Ottomo S, Oikawa M, Okada T, Shimamura H, Takemura S, Ono F, Akada M, et al: Neoadjuvant chemotherapy with gemcitabine and S-1 for resectable and borderline pancreatic ductal adenocarcinoma: Results from a prospective multi-institutional phase 2 trial. Ann Surg Oncol 20: 3794-3801, 2013

8. Moertel CG, Frytak S, Hahn RG, O'Connell MJ, Reitemeier RJ, Rubin J, Schutt AJ, Weiland LH, Childs DS, Holbrook MA, et al: Therapy of locally unresectable pancreatic carcinoma: A randomized comparison of high dose (6000 rads) radiation alone, moderate dose radiation (4000 rads+5-fluorouracil) and high dose radiation+5-fluorouracil: The gastrointestinal tumor study group. Cancer 48: 1705-1710, 1981

9. Loehrer PJ Sr, Feng Y, Cardenes H, Wagner L, Brell JM, Cella D, Flynn P, Ramanathan RK, Crane CH, Alberts SR and Benson AB III: Gemcitabine alone versus gemcitabine plus radiotherapy in patients with locally advanced pancreatic cancer: An Eastern Cooperative Oncology Group trial. J Clin Oncol 29: 4105-4112, 2011.

10. Pisters PW, Hudec WA, Lee JE, Raijman I, Lahoti S, Janjan NA, Rich TA, Crane CH, Lenzi R, Wolff RA, et al: Preoperative chemoradiation for patients with pancreatic cancer: Toxicity of endobiliary stents. J Clin Oncol 18: 860-867, 2000.

11. Krishnan S, Rana V, Janjan NA, Varadhachary GR, Abbruzzese JL, Das P, Delclos ME, Gould MS, Evans DB and Wolff RA: Induction chemotherapy selects patients with locally advanced, unresectable pancreatic cancer for optimal benefit from consolidative chemoradiation therapy. Cancer 110: 47-55, 2007.

12. Yovino S, Poppe M, Jabbour S, David V, Garofalo M, Pandya N, Alexander R, Hanna N and Regine WF: Intensity-modulated radiation therapy significantly improves acute gastrointestinal toxicity in pancreatic and ampullary cancers. Int J Radiat Oncol Biol Phys 79: 158-162, 2011.

13. Ben-Josef E, Shields AF, Vaishampayan U, Vaitkevicius V, El-Rayes BF, McDermott P, Burmeister J, Bossenberger T and Philip PA: Intensity-modulated radiotherapy (IMRT) and concurrent capecitabine for pancreatic cancer. Int J Radiat Oncol Biol Phys 59: 454-459, 2004.

14. Murphy JD, Adusumilli S, Griffith KA, Ray ME, Zalupski MM, Lawrence TS and Ben-Josef E: Full-dose gemcitabine and concurrent radiotherapy for unresectable pancreatic cancer. Int J Radiat Oncol Biol Phys 68: 801-808, 2007.

15. Nakamura A, Shibuya K, Matsuo, Y, Nakamura, M, Shiiinoki T, Mizowaki T and Hiraoka M: Analysis of dosimetric parameters associated with acute gastrointestinal toxicity and upper gastrointestinal bleeding in locally advanced pancreatic cancer patients treated with gemcitabine-based concurrent chemoradiotherapy. Int J Radiat Oncol Biol Phys 84: 369-375, 2012. 